CARNETS DE Carnets de géographes

GÉOGRAPHES.

\title{
Actualités de la géographie critique
}

Retour sur la dernière conférence internationale de géographie critique

(Francfort et Berlin, 16-20 août 2011)

Martine Drozdz, Cecile Gintrac et Sarah Mekdjian

\section{(2) OpenEdition}

Journals

Édition électronique

URL : http://journals.openedition.org/cdg/1014

DOI : $10.4000 /$ cdg. 1014

ISSN : 2107-7266

Éditeur

UMR 245 - CESSMA

Référence électronique

Martine Drozdz, Cecile Gintrac et Sarah Mekdjian, « Actualités de la géographie critique », Carnets de géographes [En ligne], 4 | 2012, mis en ligne le 01 septembre 2012, consulté le 24 septembre 2020.

URL : http://journals.openedition.org/cdg/1014 ; DOI : https://doi.org/10.4000/cdg.1014

\section{(c) $\Theta \Theta \Theta$}

La revue Carnets de géographes est mise à disposition selon les termes de la Licence Creative Commons Attribution - Pas d'Utilisation Commerciale - Pas de Modification 4.0 International. 


\section{ACTUALITES DE LA GEOGRAPHIE CRITIQUE}

Retour sur la dernière conférence internationale de géographie critique (Francfort et Berlin, 16-20 août 2011)

MARTINE DROZDZ

Université Lumière Lyon 2 / London School of Economics UMR 5600 Environnement, ville, société martinedrozdz@yahoo.fr

CECILE GINTRAC Université Paris Ouest Nanterre La Défense EA 375 - Laboratoire de géographie comparée des Suds et des Nords (GECKO) cecile.gintrac@gmail.com

SARAH MEKDJIAN

Maître de conférence à l'Université Pierre Mendès-France Grenoble 2

UMR PACTE sarah.mekdjian@upmf-grenoble.fr

La 6e conférence internationale de géographie critique s'est déroulée à Francfort du 16 au 20 août 2011. Pendant quatre jours, environ trois cents participants venus de nombreux pays se sont réunis pour débattre autour des " crises " (Causes, Dimensions, Réaction), thématique incontournable au moment même où la crise des dettes souveraines européennes s'intensifiait. Cette conférence fut la Gème organisée depuis la création de cet évènement en 1997 à Vancouver, et la seconde à être organisée en Europe, et offrait un éventail varié des approches et des thématiques qui se retrouvent sous la bannière des géographies critiques. Avant de détailler les thèmes et les approches explorées pendant la conférence, nous nous proposons de revenir sur la genèse et le contexte de création de cet événement.

\section{Aux origines du groupe international de géographie critique : un positionnement} militant et contestataire.

La première conférence de géographie critique à la fin des années 1990 naît de l'impératif, pour les géographes anglophones, de répondre à un double contexte problématique : les agissements de la firme Shell au Nigeria, l'un des principaux mécènes de la conférence annuelle des géographes britanniques (RGS-IBG) et la crise économique qui touche l'Asie du Sud-Est et les pays émergents (Argentine, Russie, 
Brésil), événement qui met un frein (temporaire) à l'enthousiasme des chantres de la mondialisation néolibérale et permet l'expression de voix critiques.

Revenons sur le premier événement déclencheur. L'exécution de neuf activistes Ogoni dont l'écrivain Ken Saro-Wiwa par le régime militaire de Lagos en 1995, déclenche une vague de contestation chez les géographes anglophones, en particulier Britanniques, dont la réunion annuelle est sponsorisée par la firme Shell, soutien financier du régime militaire nigerian et possiblement directement impliqué dans ces crimes (Berg, 2009). Les universitaires britanniques (membre du Institute of British Geographers - IBG) mènent alors une campagne en faveur de l'interruption du mécénat de Shell de leur conférence annuelle. La motion est unanimement acceptée par les universitaires du IBG et massivement rejetée par les membres de la Royal Geographical Society (RGS). Face à cette situation, les géographes britanniques (fédérés autour du Critical Geography Forum Online modéré par Joe Painter) proposent de créer une conférence alternative à la réunion annuelle de la RGS-IBG. Ce forum devient un espace de discussion pour les géographes anglophones, qui proposent alors d'organiser à Vancouver la première Conférence Internationale de Géographie Critique.

Chronologie du groupe international de géographie critique

\begin{tabular}{|c|c|}
\hline 1980 & Premier symposium nordique sur la géographie critique. \\
\hline 1982 & $\begin{array}{l}\text { Publication de la série de livres édités par Macmillan dans la collection Critical } \\
\text { Human Geographx. }\end{array}$ \\
\hline $\begin{array}{l}\text { Années } \\
1990\end{array}$ & $\begin{array}{l}\text { Fusion de la Royal Geagrophical Society et du Institute of British Geographer et } \\
\text { obtention du mécénat de la firme Shell. }\end{array}$ \\
\hline 1994 & Mini-conférence de géographie critique à Çincinatti (Etats-Unis). \\
\hline 1995 & $\begin{array}{l}\text { - Neuf membres du groupe des Ogonis sont exécutés par le gouvernement Nigérian. } \\
\text { L'implication de la compagnie Shell, très présente dans le delta du Niger, dans cette } \\
\text { exécution est évoquée. } \\
\text { - Création du forum de géographie critique, conçu et revendiqué comme une } \\
\text { alternative à la RGS-IBG. } \\
\text { - Motion anti-Shell rejetée au moment e la tenue de l'annuelle conférence de la RGS- } \\
\text { IBG. }\end{array}$ \\
\hline 1997 & $\begin{array}{l}\text { - Inauguration de la première conférence internationale de géographie critique à } \\
\text { Vancouver (Canada). } \\
\text { - Création du comité d'organisation des conférences internationales de géographie } \\
\text { critique, sur la base exclusive du volontariat. }\end{array}$ \\
\hline 2000 & Deuxième conférence internationale de géographie critique à Taegu (Corée du Sud). \\
\hline 2002 & Troisième conférence internationale de géographie critique à Békéscaba (Hongrie). \\
\hline 2005 & Quatrième conférence internationale de géographie critique à Mexico (Mexique). \\
\hline 2007 & Cinquième conférence internationale de géographie critique à Mumbai (Inde). \\
\hline 2011 & Sixième conférence internationale de géographie critique, Francfort (Allemagne). \\
\hline
\end{tabular}

Source : D'après Berg, 2009 
Cette première conférence est l'occasion de fédérer ou de faire se rencontrer différents mouvements de "géographie critique ", émergeant dans des contextes universitaires variés depuis les années 1980 et qui désignent un large spectre d'approches et d'objets. Ces approches ont pour caractéristique commune la volonté de dénoncer et déconstruire les situations de domination, de révéler les relations spatiales inégalitaires, dans le but de transformer les structures qui les produisent ( $a$ broad coalition of left-progressive approaches to the study of Geography [...] linked by a shared commitment to a broadly conceived emancipatory, progressive social change, and the use of a range of critical socio-geographic theories - Berg, 2009).

\section{2. "A world to win !"}

C'est ce slogan, résolument optimiste, qui introduit la déclaration d'intention du groupe international de géographie, déclinée en douze points. II nous semble que cette déclaration s'articule autour de trois idées centrales, qui permettent de mieux appréhender les critères de définition de la géographie critique :

- la volonté d'analyser, pour mieux les dénoncer, la diversité des formes de domination : "capitalist exploitation; oppression on the basis of gender, race and sexual preference; imperialism, national chauvinism, environmental destruction" (Comité d'organisation de la conférence). Cette approche semble d'autant plus nécessaire que la géographie, en tant que discipline, est longtemps apparue comme un outil au service des puissants : "the discipline has long served colonial, imperial and nationalist ends by generating the ideological discourses that help to naturalize social inequality" (ibid.).

- I'affirmation d'un engagement auprès des mouvements sociaux : "we join with existing social movements outside the academy aimed at social change" (ibid.). II s'agit de dépasser les frontières du monde académique pour intervenir dans l'espace public et soutenir certaines luttes.

- la nécessité de créer un réseau alternatif de recherche en géographie, dans un contexte de privatisation croissante des savoirs et de l'enseignement. "We are critical because we refuse the self-imposed isolation of much academic research, believing that social science belongs to the people and not the increasingly corporate universities" (ibid.).

Il s'agit donc d'une approche qui se veut alternative, tant par les savoirs produits que par les conditions d'exercice d'une recherche dont les effets ne doivent pas se limiter au monde académique.

\section{Retour sur Francfort}

L'analyse du déroulement de cette conférence nous permet de répondre à la question suivante : qu'est-ce qu'une conférence de géographie critique ? En quoi celle-ci diffèret-elle des conférences classiques?

\section{a) L'espace au prisme de la crise}

La conférence était structurée autour de la notion de crise, et dix thèmes guidaient les interventions:

- la crise économique, financière et fiscale ;

- la crise urbaine ;

- la crise écologique ;

- $\quad$ les subjectivités en crise ?; 
- les mouvements d'opposition;

- $\quad$ la géopolitique, la biopolitique et les espaces critiques du politique ;

- $\quad$ les mobilités en crise ;

- $\quad$ l'université / la géographie en crise ;

- la question de la traduction ;

- l'Europe et « ses autres".

En ce sens, le fil directeur de la crise avait pour ambition de rendre les savoirs produits véritablement opératoires (comment comprendre la crise et ses effets ? Quels moyens d'action permettent d'y répondre ? ).

Le premier thème a ainsi permis d'entendre plusieurs communications sur les manifestations, les causes et les conséquences de la crise du capitalisme financier depuis 2008 et celle des dettes souveraines européennes, avec quelques interventions sur les transformations neolibérales de l'Etat post-keynésien, ou de " l'Etat keynésien privatisé ", comme le qualifie l'économiste autrichienne Brigitte Young.

La crise urbaine a occupé 24 sessions sur la centaine de panels proposés au total, et donné à voir une certaine diversité géographique (avec des études de cas situées au Canada, en Suède, Australie, Etats-Unis, Royaume Uni, Afrique du Sud, Slovaquie, Allemagne, Pays-Bas, Taiwan, Hong-Kong, Brésil, Chili, Colombie, Bengladesh, Palestine...) et thématique : les crises des crédits immobiliers et leurs géographies, la privatisation des services urbains - réseaux de distribution de l'eau, logements - et leurs conséquences sociales, les usages du concept de "neighborhood" dans les politiques des gestion de l'espace urbain, la marchandisation des espaces publics et dans ce cadre, le rôle joué par les TICes, et plus généralement, les pratiques d'exclusion des espaces publics, via l'analyse d'opérations immobilières ou la mise en place de politiques sécuritaires.

Le thème des luttes sociales était lui aussi majoritairement situé dans un contexte urbain, avec des positions qui examinaient les possibilités/horizons contestataires, voire révolutionnaires, les luttes locales contre l'urbanisme néo-libéral, mais aussi les modes d'organisation des groupes marginalisés (travailleur.se.s du sexe, sweatshops, vendeurs de rue). Deux sessions étaient centrées sur la production des biens communs urbains (urban commons).

La géographie rurale et l'environnement, particulièrement peu présents, partageaient une session commune, pour une quinzaine de présentations au total où se mêlaient crise alimentaire, changement climatique, dynamiques sociales des espaces ruraux et mobilisations environnementales.

Le thème "subjectivités en crise" proposait un ensemble de communications ayant pour point commun de s'interroger sur les processus d'identification liés au genre, aux mouvements queer, aux situations de handicap (par exemple les spatialités des personnes handicapées en Hongrie), etc. Le rôle de l'espace dans la construction mais aussi la marginalisation de ces "minorités". II comportait également deux sessions exclusivement urbaines, centrées sur le thème "droit à la ville", exploré du point de vue de certains groupes (femmes, lesbiennes gay bi et trans).

Le thème consacré à l'analyse des biopolitiques (forme d'exercice du pouvoir qui porte sur la gestion du vivant, des populations, et de leur corps) offrait une succession de sessions très cohérentes. Une présentation d'études de cas de biopolitiques contemporaines, dans des contextes géographiques variés (Afghanistan, Chine) était suivie par une analyse plus historique des biopolitiques réalisées au Japon. La session 
suivante donnaient à voir les résistances que des groupes locaux marginalisés ou menacés par ces politiques peuvent mettre en place (en Amérique latine) quand le dernier panel se concentrait spécifiquement sur la territorialisation des programmes biopolitiques contemporains. On notera qu'un dernier panel interrogeait les spatialités de l'Holocauste (Holocaust spatialities).

La condition des migrants internationaux, dans des contextes de durcissement des politiques publiques d'encadrement et de contrôle des flux migratoires était au coeur de la question des "mobilités en crise". Plusieurs études de cas soulignaient la spécificité des contextes (mobilités forcées des Roms en Italie ou encore mobilités urbaines à travers l'analyse comparées des systèmes de transport de Bogota et Mexico). Les panels du thème "I'Europe et ses autres" complétaient ces questions en interrogeant les processus de renforcement des frontières extra-communautaires.

Les sessions centrées sur l'analyse de la crise de l'Université offraient un espace de dialogue et de partage d'expériences pour des enseignants et des chercheurs engagés dans des institutions dont le rôle et le fonctionnement ont été bouleversés par la diffusion de l'idéologie néolibérale. La plupart des panels cherchaient à pointer les effets de la privatisation de l'Université et la commodification ${ }^{1}$ du savoir. La précarisation de la force de travail universitaire et la marginalisation des femmes occupaient deux sessions. L'analyse des conditions de production des savoirs font partie d'une approche réflexive, constituant sans doute l'un des apports majeurs de la géographie critique. C'est également dans cette perspective que la question du rôle de traduction a été posée. L'hégémonie de l'anglais comme langue de production et de diffusion des savoirs a occupé une partie des discussions organisées de manière volontairement plurilingues par les participants (la communication de Claire Hancock sur la figure du traducteur était en partie bilingue, en français et en anglais). Un des enjeux fut de penser les modalités d'une circulation des savoirs, qui limiterait les effets de domination, de normalisation et de marginalisation des pensées en langues dites " étrangères $"$.

En somme, si l'on examine l'ensemble des communications sans prendre en compte le découpage en dix thématiques proposées, force est de constater que la géographie critique est le plus souvent une géographie urbaine : plus d'une centaine de communications partaient d'exemples urbains.

C'est ce que confirme le nuage de mots réalisés à partir des titres des interventions.

\section{Nuage de mots réalisé à partir des titres des interventions}

\footnotetext{
"Par "commodification", on entend ici la transformation du savoir en marchandise, en " commodité » qui peut s'échanger sur le marché mondial des connaissances, libéré de son contexte de production et de réception initiaux.
} 


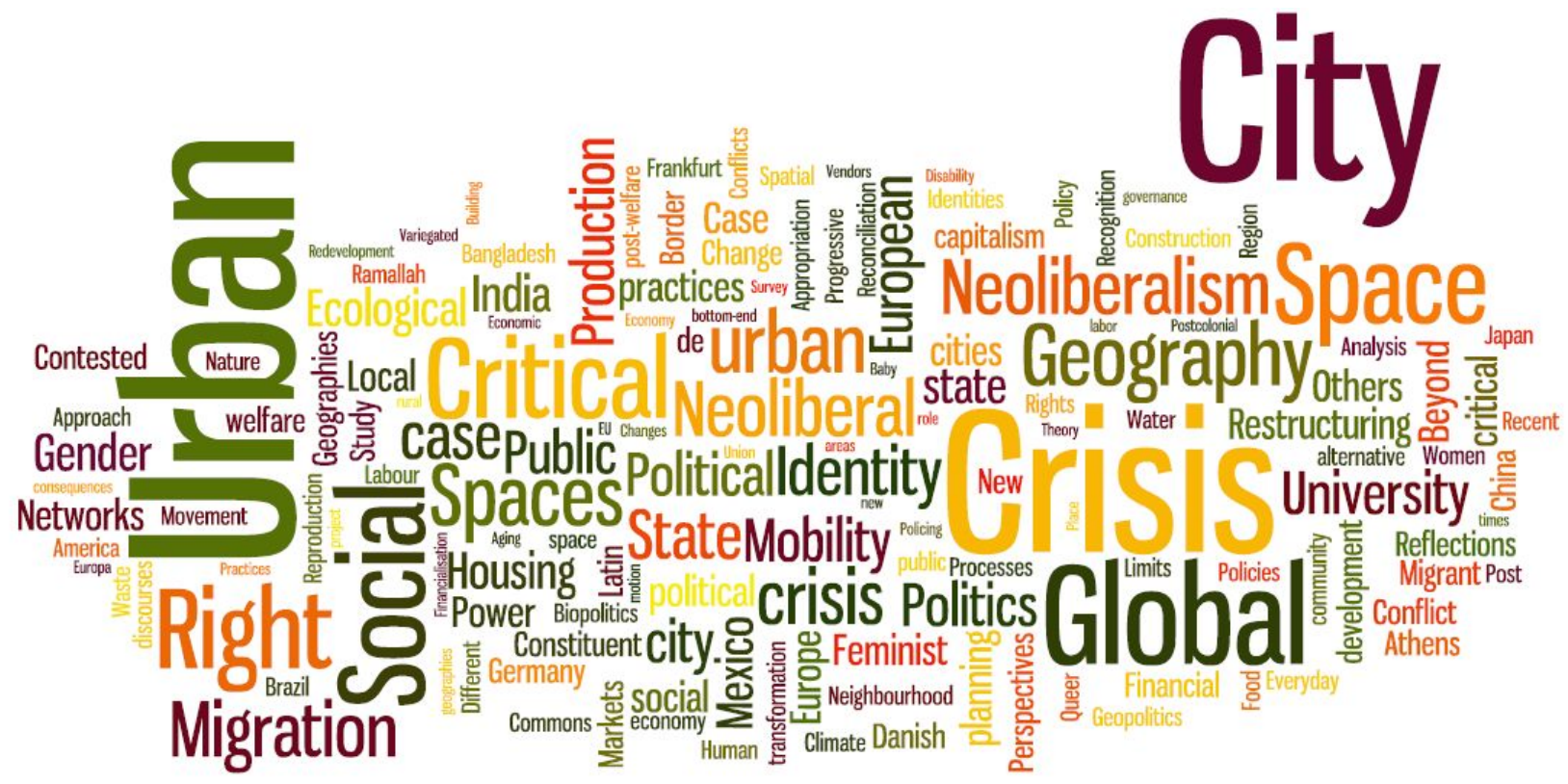

Au-delà des objets privilégiés par la géographie critique, une méthodologie spécifique se dégage t-elle?

\section{b) Pensées critiques, méthodes critiques ?}

$\mathrm{Si}$ les thématiques employées permettaient de retrouver les objets chers aux géographes critiques et radicaux (genre, mouvements sociaux et mobilisation collectives, gentrification), on peut souligner que la question de la méthodologie a globalement été peu interrogée. La plupart des présentations correspondaient en fait à des études de cas, qui pouvaient donner lieu parfois à une déconstruction des discours dominants. C'est d'ailleurs ce que soulignait Peter Marcuse, figure des critical urban studies dans la session finale sur la ville, en soulignant la pression croissante au sein de l'Université pour produire des "faits". La multiplication des études de cas, si pertinentes soient-elles, est susceptible d'empêcher les généralisations théoriques ("Too much empircal studies prevents critical thinking", Marcuse). La place prépondérante des études empiriques est-elle le signe d'un déficit de théorisation ou le résultat d'un choix délibéré de poser les bases d'un savoir ancré dans la pratique? II semblerait que les participants considèrent davantage l'approche critique par le choix de leurs objets empiriques et les références à des auteurs reconnus dans ce champ (Henri Lefebvre, David Harvey, Michel Foucault, Neil Smith, Ecole de Francfort,) que par des innovations théoriques et méthodologiques.

Dans ce contexte, la question des échelles a, par exemple, finalement été peu interrogée. II semblerait pourtant pertinent de s'en emparer davantage. A quelle échelle penser les formes de domination? A quelle échelle penser ou pratiquer les luttes?

Deux jours de voyage de terrain à Berlin concluaient la conférence, organisés par des universitaires allemands engagés dans divers mouvements sociaux urbains. Un choix d'une dizaine d'excursions a permis de prolonger les débats ouverts à Francfort et de recontrer des activistes berlinois, tels que des associations LGBT en lien avec l'immigration turque, des militants engagés dans la lutte contre la multiplication des caméras de surveillance ou encore des anciens squatteurs du quartier de Kreuzberg. 
C'est là un exemple paticulièrement original et appréciable d'un savoir partagé, en construction avec les acteurs de terrain.

A Francfort, comme à Berlin, les débats ont fait émerger un ensemble des notions qui nous semblent particulièrement pertinentes pour comprendre les enjeux contemporains d'une géographie critique.

\section{c) Eclairage de quelques concepts qui ont retenu notre attention}

- commodification / décommodification des biens urbains : plusieurs intervenants évoquaient la " décommodification " des biens urbains comme une régulation possible des effets les plus néfastes de la gentrification. Rappelons que le terme de commodification est un néologisme anglais traduit le plus souvent par "marchandisation". Par opposition, la décommodification désigne le processus par lequel des populations, des espaces, des biens matériels ou immatériels peuvent sortir des logiques du marché.

Le contexte berlinois se prêtait particulièrement à une réflexion sur ce couple de notions. La ville donne à voir tout un éventail de possibilités de logement hors des lois du marché - coopératives, squats, logements sociaux. Ces alternatives sont menacées par des politiques urbaines liées à la crise fiscale, qui visent à les marchandiser. Ces réflexions rejoignaient les débats initiés à Francfort sur les politiques de régulation et de gestion des biens urbains collectifs (urban commons) soumis à la généralisation des logiques de privatisation. Plus généralement, c'est la question de la nature et des propriétés des biens produits en dehors du marché qui encadre ces réflexions, dans le prolongement des analyses récentes de l'économie politique ${ }^{2}$.

- Le droit à la ville (Right to the city) : I'ombre d'Henri Lefebvre planait sans nul doute sur Francfort et Berlin pendant cette semaine. Quatre panels étaient consacrés au droit à la ville auxquels il faut ajouter les nombreuses présentations qui mentionnaient ce concept lefevbrien dans le titre. Le droit à la ville est devenu manifestement un outil de réflexion sur l'émancipation au même titre qu'un outil de mobilisation. Une session était ainsi consacrée aux pratiques politiques des géographes critiques au sein de Right to the City Alliance ("Political Practice of Critical geographers within the Right the the City Alliance"). Plusieurs participants de la conférence revendiquaient une activité au sein de ce mouvement qui s'est structuré à partir de 2007 depuis les États-Unis.

La conférence internationale de géographie critique de Francfort, comme les précédentes éditions, avait pour principal objectif d'offrir la possibilité d'un échange entre des géographes qui partagent la volonté de participer à la transformation du monde qu'ils décrivent. Le fait qu'il ne s'agisse pas d'un courant identifié comme tel, mais davantage d'un positionnement prenant des formes variées, rend cette "plateforme" nécessaire pour mettre en commun les savoirs produits. Cependant on peut noter que le format de la conférence n'est pas si différent des autres rencontres

\footnotetext{
${ }^{2}$ Pour la question de la nature et des propriétés des biens produits en dehors du marché voir en particulier les réflexions de Yochai et Benkler (Benkler, 2009), et en particulier la préface de la traduction française (Agrain, 2009). Pour la question de la gouvernance des commons, voir les travaux d'Elinor Ostrom, prix Nobel d'économie en 2009.
} 
académiques, si ce n'est (et c'est assez rare pour le noter) la convivialité assumée et la jeunesse des participants.

Quinze ans après la première conférence, l'optimisme ne peut cependant qu'être mesuré et les critiques formulées par Neil Smith et Carole Desbiens (Smith and Desbiens, 1999) dans un article de référence, revenant sur les ambitions de ces rencontres, restent en grande partie valides :

- la surreprésentation de quelques pays européens et nord-américains, malgré les efforts de redistribution et de soutien financiers (sponsoring de la revue Antipode) en vue de favoriser la mobilité des chercheurs dont les ressources sont limitées ;

- la faiblesse des liens avec l'activisme extra-universitaire, alors qu'il s'agit d'un des fondements de de la Déclaration d'intention, malgré les efforts déployés lors du voyage de terrain à Berlin ;

- la question délicate de la traduction, mais plus généralement, celle de la domination de l'anglais, a été posée à juste titre. Dans ce cadre, le refus de certains universitaires anglophones de rendre accessible le contenu de leur présentation à un public nonanglophone était regrettable. Le comité d'organisation avait pourtant multiplié les appels à utiliser les diaporamas de manière à favoriser la visibilité et la compréhension des communications.

Dans un contexte de précarisation et de mise en concurrence croissantes des chercheurs, cet espace d'échanges véritablement convivial, organisé avec l'aide de la jeune génération de la géographie critique allemande, offre une bouffée d'air frais dans le monde de la géographie.

\section{Liens}

Site officiel du groupe international de géographie critique (en construction) : http://internationalcriticalgeography.org/

Liste de diffusion : www.jiscmail.ac.uk/ICGG

Revue ACME (An International E-Journal for Critical Geographies): http://www.acmejournal.org/

Right to the City Alliance : http://www.righttothecity.org/WhoWeAre.html

\section{Bibliographie}

Nous tenons à remercier les Professeur-es Lawrence Berg, Alex Demirovic, Heide Gerstenberger et Joe Painter pour leur aide dans la documentation de cet article.

Agrain P. (2009) "Liberté, justice, développement humain", La richesse des réseaux, pp.

http://presses.univ-

lyon2.fr/ATTACH/0000000811/OTHERTEXT/0000002190.pdf

Benkler Y. (2009) La richesse des réseaux: Marchés et libertés à l'heure du partage social, PUL, 2009, 603 p.

Berg L.D. (2009) "Critical Human Geography” in Encyclopedia of Human Geography

International Critical Geography Group, "Statement of Purpose", http://internationalcriticalgeography.org/statement-of-purpose/, consulté le 01 décembre 2011.

Marcuse P. (2011) "Urban Crisis. What follows?", extrait du débat, 6e conférence de géographie critique, Francfort. 
Painter J., (2003), 'The International Critical Geography Group' (texte de travail) publié par le Department of Geography, University of Durham, http://www.dur.ac.uk/j.m.painter/ICGG.htm

Smith N., Desbiens C. (1999) "The International Critical Geography Group: forbidden optimism?," Environment and Planning D, 17, 4, p.p.379-382. 